\title{
EXCHANGE MAGNETOSTRICTION IN DISORDERED 2D ISING FERROMAGNETS
}

\author{
B. Kawecka-Magiera, A. MaKsymowicz, K. Kulakowski and A. Lenda \\ Faculty of Physics and Nuclear Techniques, AGH \\ Al. Mickiewicza 30, 30-059 Kraków, Poland
}

\begin{abstract}
We investigate volume and shape magnetoelastic strains of a $2 \mathrm{D}$ anisotropic Ising alloy with partial atomic order. Three possible origins of shape strain are distinguished: (i) anisotropy of elastic constants, (ii) anisotropy of magnetic bonds and (iii) anisotropy of the spatial derivatives of magnetic bonds. None of these mechanisms vanishes above the Curie temperature. Equilibrium strains are expressed in terms of anisotropic spin-spin nearest-neighbours correlation functions. These functions are calculated against temperature, both in ferromagnetic and paramagnetic phase. Shape strain decreases monotonously with temperature for the mechanisms (i) and (iii), and has a maximum near the Curie temperature for the case (ii). The results may be relevant for multilayer magnetic systems.
\end{abstract}

PACS numbers: $75.80 .+q, 05.50 .+q$

\section{Introduction}

A two-dimensional $S=1 / 2$ Ising system of a substitutional alloy $\mathrm{A}_{c} \mathrm{~B}_{1-c}$ with anisotropic nearest-neighbour interactions is studied as a possible source of magnetostriction in the system. The Hamiltonian is assumed of the form

$$
\mathcal{H}=-\frac{1}{2} \sum_{i, j} J_{i j} S_{i} S_{j}
$$

where the sum is over $(i, j)$ sites which are nearest neighbours. The exchange integrals may take on one of the six values, $J_{i, j}=J_{a a}^{\mu}, J_{a b}^{\mu}, J_{b b}^{\mu}$, where $\mu=x$ or $y$ depending on the pair orientation along $\mu=x$ or $y$-axes, and the type $(a a),(a b)$ or $(b b)$ of atomic pair located on $(i, j)$ site.

In this paper we are interested in anisotropic linear strains which result in our case from isotropic exchange interaction. The strength of the coupling, however, depends on the pair orientation. Some valuable information may be obtained from an analysis of the Ising model, where only one component of spins is taken into account. Within such a model, the origin of magnetoelastic coupling is the distance dependence of exchange integrals $J_{i, j}$.

The description of the magnetoelastic coupling follows the Callen and Callen theory [1]. Their approach is based on magnetoelastic energy expressed in terms of 
invariants constructed from products of polynomials of different degree of elastic strains and magnetization direction cosines. Then the strains may be obtained from the condition that total energy, which depends on the thermal average of the spin-spin correlation functions, reaches minimum. As a result, we get temperature dependence of volume and shape magnetoelastic coefficients. In this paper, the spin-spin correlation functions are studied for system with short-range atomic ordering, and for a possible directional dependence of exchange integrals.

\section{The model}

The model is limited, for simplicity, to the case of shape strain parallel to the direction of magnetization. This assumption means that anisotropy of elastic constant is uniaxial, parallel to magnetic anisotropy, and nondiagonal elements of strain tensor $\varepsilon_{i j}$ are excluded. Within the Ising model, we consider the spin-spin correlation function $\left\langle S_{i}^{z} S_{j}^{z}\right\rangle$. According to the Callen theory [1], macroscopic magnetoelastic energy $U_{\mathrm{me}}$ consists of two magnetoelastic terms with $B_{\mathrm{v}}$ and $B_{\mathrm{s}}$ constants corresponding to the volume and shape contributions, respectively. We have

$$
U_{\mathrm{me}}=\left(B_{\mathrm{v}} \eta_{\mathrm{v}}+B_{\mathrm{s}} \eta_{\mathrm{s}}\right)\left\langle S_{i}^{z} S_{j}^{z}\right\rangle
$$

Corresponding strains $\eta$ are

$$
\begin{aligned}
& \eta_{\mathrm{v}}=\left(\varepsilon_{x x}+\varepsilon_{y y}\right) / \sqrt{2}, \\
& \eta_{\mathrm{s}}=\left(\varepsilon_{x x}-\varepsilon_{y y}\right) / \sqrt{2},
\end{aligned}
$$

and the elastic energy $U_{\mathrm{el}}$ expressed in terms of $\eta^{\prime} s$ is [2]

$$
U_{\mathrm{el}}=\frac{1}{2} c_{11}^{\alpha} \eta_{\mathrm{v}}{ }^{2}+\frac{1}{2} c_{22}^{\alpha} \eta_{\mathrm{s}}{ }^{2}+c_{12}^{\alpha} \eta_{\mathrm{v}} \eta_{\mathrm{s}}
$$

where $c_{i j}^{\alpha}$ are elastic constants of the system. Minimizing $U_{\mathrm{el}}+U_{\mathrm{me}}$ with respect to the strains $\eta$, one gets

$$
\begin{aligned}
& \eta_{\mathrm{v}}=\frac{1}{\Delta}\left[c_{22}^{\alpha} \frac{\partial U_{\mathrm{me}}}{\partial \eta_{\mathrm{v}}}-c_{12}^{\alpha} \frac{\partial U_{\mathrm{me}}}{\partial \eta_{\mathrm{s}}}\right] \\
& \eta_{\mathrm{s}}=\frac{1}{\Delta}\left[c_{12}^{\alpha} \frac{\partial U_{\mathrm{me}}}{\partial \eta_{\mathrm{v}}}-c_{11}^{\alpha} \frac{\partial U_{\mathrm{me}}}{\partial \eta_{\mathrm{s}}}\right]
\end{aligned}
$$

where

$$
\Delta=c_{12}^{\alpha^{2}}-c_{11}^{\alpha} c_{22}^{\alpha}
$$

The magnetoelastic energy is just the thermal average of the Ising energy

$$
U_{\mathrm{me}}=-\frac{1}{2}\left\langle\sum_{i, j} J_{i j} S_{i}^{z} S_{j}^{z}\right\rangle
$$

where $S^{z}= \pm 1$. The average $(\ldots)$ is calculated by means of computer simulation technique. The derivatives of energy $U_{\text {me }}$ with respect to strains are to be expressed by the derivatives of exchange integrals $J$ with respect to the distance. The latter depends on atomic ordering and on direction of atom pairs.

Since the exact solution for the energy $U_{\text {me }}$ given by Eq. (9) exists for $J_{a a}=$ $J_{a b}=J_{b b}$ and for perfect atomic order, we can obtain the magnetoelastic energy contribution in this case. However, if the integrals $J_{i j}$ and their spatial derivatives 
are different for atomic pairs parallel to $x$ - or $y$-axis, we expect magnetic anisotropy energy which is a necessary condition for magnetoelasticity. Let us denote these integrals by $J_{x}$ and $J_{y}$, respectively. As we know [3], correlation function $\left\langle S_{i}^{z} S_{j}^{z}\right\rangle$ depends both on $J_{x}$ and $J_{y}$.

From now on, we approximate the magnetoelastic energy (9) per each atomic pair as

$$
U_{\mathrm{me}}=-\bar{J}_{x}\left\langle S_{i}^{z} S_{j}^{z}\right\rangle_{x}-\bar{J}_{y}\left\langle S_{i}^{z} S_{j}^{z}\right\rangle_{y},
$$

where the dashed constants $J$ are the exchange integrals averaged over local atomic configurations for $x$ - and $y$-directions, respectively.

Within approximation (10), we can express volume (or surface) and shape strains as linear combinations of the two correlation functions $\left\langle S_{i}^{z} S_{j}^{z}\right\rangle_{x, y}$

$$
\begin{aligned}
& \eta_{\mathrm{v}}=\frac{N}{\Delta \sqrt{2}}\left[\left(c_{22}^{\alpha}-c_{12}^{\alpha}\right) \bar{J}_{x} f_{x}+\left(c_{12}^{\alpha}+c_{22}^{\alpha}\right) \bar{J}_{y} f_{y}\right] \\
& \eta_{\mathrm{s}}=\frac{N}{\Delta \sqrt{2}}\left[\left(c_{11}^{\alpha}+c_{12}^{\alpha}\right) \bar{J}^{\prime}{ }_{y} f_{y}-\left(c_{11}^{\alpha}-c_{12}^{\alpha}\right){\overline{J^{\prime}}}_{x} f_{x}\right]
\end{aligned}
$$

where the prime denotes spatial derivatives, and

$$
f_{x, y}=\left\langle S_{i}^{z} S_{j}^{z}\right\rangle_{x, y} \text {. }
$$

\section{Numerical results and discussion}

The thermal averages $(\ldots)$ are calculated by means of the standard Monte Carlo technique when spins are sequentially updated using the local information of the surrounding spins. We performed calculations of the Curie temperature $T_{\mathrm{c}}$, magnetization $m(T)$ and spin-spin correlation $\left\langle S_{i}^{z} S_{j}^{z}\right\rangle$. The correlations $\langle S S\rangle_{x}$ and $\langle S S\rangle_{y}$ in two directions, averaged over all types of atom pairs, were extracted for neighbouring spins. The net size used for simulation was $512 \times 512$ sites, with nearest-neighbours interactions. Full periodic boundary conditions were applied.

As it is seen from Eqs. (11), (12), there are three possible mechanisms of non-vanishing shape strain $\eta_{\mathrm{s}}$ :

(i) anisotropy in elastic constants, i.e. for $c_{12}^{\alpha}$ different from zero,

(ii) anisotropy in spin-spin correlations $\langle S S\rangle$ induced by anisotropy in magnetic bonds $J$, and

(iii) anisotropy of the distance derivatives of magnetic bonds $J^{\prime}$.

For cases (i) and (iii), the temperature dependence of $\eta_{\mathrm{s}}$ follows the temperature dependence of isotropic correlation function, $f_{x}=f_{y}$. For case (ii), the shape strain is proportional to the difference of two anisotropic correlation functions $f_{x}-f_{y}$. In practice, one expects that mechanisms (ii) and (iii) are correlated. Then monotonous reduction of the shape strain with temperature, if found experimentally, should be assigned to case (i). This particular mechanism may become active when, for example, an amorphous film is deposited on crystalline substrate with anisotropic elastic constants. If mechanisms (ii) and (iii) are dominant, we should observe a superposition of anisotropy of bonds and their derivatives. The weights of two contributions may depend on the material. 
For calculations we used different exchange integrals $J_{x} / J_{y}=0.4$ in the $x$ and $y$ - directions, as a driving force of magnetic anisotropy. To test sensitivity of the magnetic system to atomic ordering, we did the calculations for exchange integrals $J_{i ; j}$ which were chosen different for different atomic pairs in a binary alloy of $a$ and $b$ atoms. Stoichiometric concentration $1 / 2$ of both kinds of atoms makes perfectly ordered case a compound of a chessboard-like-form where only $(a, b)$ pairs are present, and so $J_{a, a}$ and $J_{b, b}$ parameters are then dummy. For disordered case, however, all $J$ 's play a role and magnetic properties may differ. We assumed for numericals $J_{a, a}=0.5 J, J_{a, b}=J, J_{b, b}=2 J$. Main results of spin-spin correlation function $\langle S S\rangle$, a veraged over all types of atom pairs and in both $x$ - and $y$-directions, against reduced temperature $T / T_{\mathrm{c}}$ are shown in Fig. 1. It is seen that non-zero correlations exist well above the Curie temperature $T_{\mathrm{c}}$. For comparison, the molecular field results, when $\langle S S\rangle=\langle S\rangle\langle S\rangle$ and so it vanishes above $T_{\mathrm{c}}$, are also displayed. Alloying is a less significant factor as the lines corresponding to atomic order/disorder cases are close to each other. Figure 2 shows the difference $\langle S S\rangle_{y}-\langle S S\rangle_{x}$ for the two directions, which is responsible for the magnetic energy anisotropy. Again, alloying is not crucial. The anisotropy, however, is clearly seen.

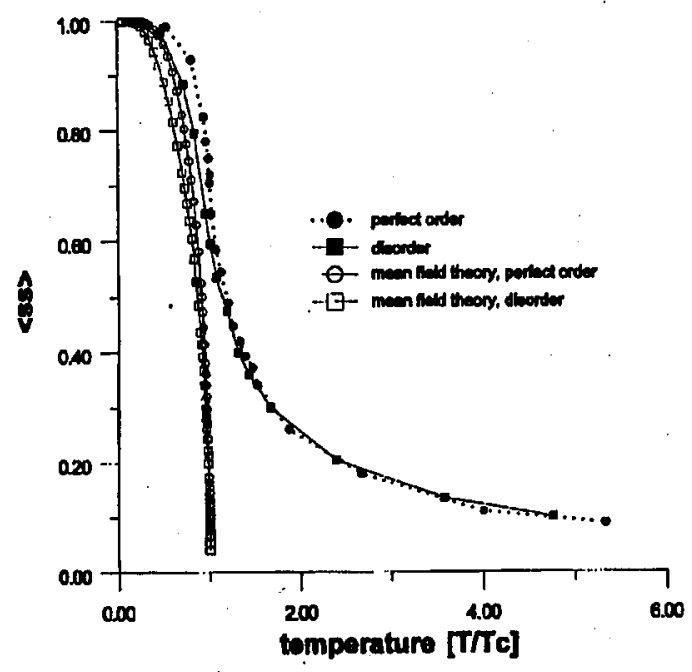

Fig. 1. Temperature dependence of the correlation function $\langle S S\rangle$.

In conclusion, we have discussed anisotropic strains of magnetic systems, which may be due to the existence of isotropic exchange with some anisotropy in model parameters. We may indicate three possible mechanisms of the effect; two of them yield a monotonous temperature dependence of the strain, the third one offers a maximum near the Curie temperature. Both volume and anisotropic strains depend on spin-spin correlation functions. These functions were reproduced by computer simulation of a two-dimensional Ising alloy. We believe that the obtained results may be relevant for some magnetic multilayers, where deflection is absent and the assumptions on the symmetry (or lack of the symmetry) can be 


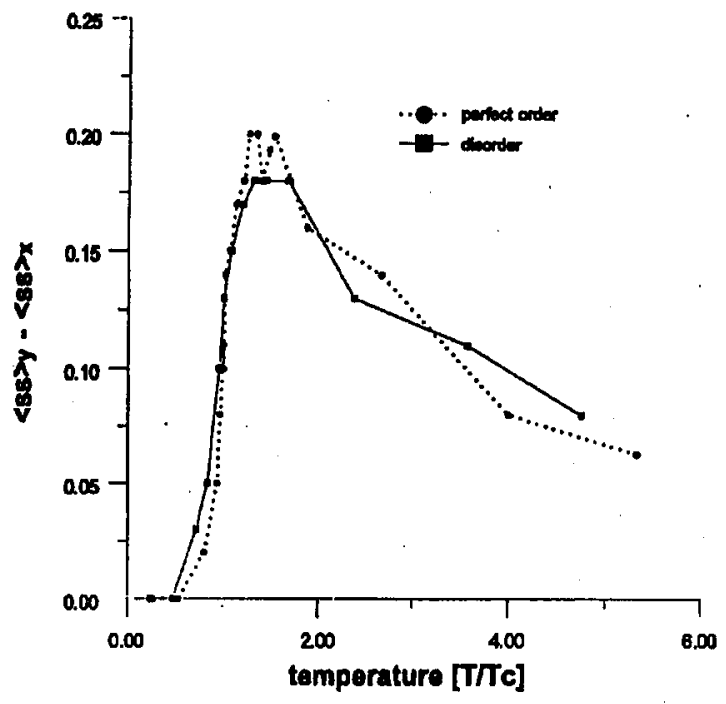

Fig. 2. Anisotropy seen as the difference $\langle S S\rangle_{y}-\langle S S\rangle_{x}$ in correlation functions.

justified and that the proposed anisotropic exchange integrals may be thought of as a candidate for magnetostrictive mechanism. The anisotropy of bonds can also be induced, for example, by annealing of a multilayer system in the presence of magnetic field below the Curie temperature.

\section{References}

[1] E.R. Callen, H.B. Callen, Phys. Rev. 139, A455 (1965).

[2] A.Z. Maksymowicz, J. Magn. Magn. Mater. 53, 221 (1985).

[3] L. Onsager, Phys. Rev. 65, 117 (1944). 